\title{
Economical Phase-Covariant Cloning of Qudits
}

\author{
Francesco Buscemi* Giacomo Mauro D’Ariano $\dagger$ and Chiara Macchiavelld \\ QUIT Group, Dipartimento di Fisica "A. Volta", \\ Università di Pavia, via Bassi 6, I-27100 Pavia, Ital\$\$
}

(Dated: July 14, 2004)

\begin{abstract}
We derive the optimal $N \rightarrow M$ phase-covariant quantum cloning for equatorial states in dimension $d$ with $M=k d+N, k$ integer. The cloning maps are optimal for both global and single-qudit fidelity. The map is achieved by an "economical" cloning machine, which works without ancilla.
\end{abstract}

PACS numbers: 03.65.-w 03.67.-a

\section{INTRODUCTION}

In quantum information the study of optimal cloning machines is a focus of interest since, by definition, cloning is synonymous of multiplexing quantum information, which has limitations in principle by the no-cloning theorem [1, 2]. In the large variety of proposals for optimal cloners, the fidelity of the machine depends on the choice of input states, with the machine often working in a covariant way, producing "rotated" clones from rotated inputs. In particular, the case of $\mathbb{S U}(d)$ covariance corresponds to universal cloning [3, 4, 5], with equal fidelity for all unitarily connected states, e. g. for all pure states. Clearly, by taking smaller input set of states the cloning performance can be improved, e. g. for smaller covariance groups. Also in connection with the eavesdropping strategies in BB84 quantum cryptography [6], the phase-covariant cloning of equatorial states has been extensively studied for qubits [7, [8], and more generally qudits [9], the latter also with the motivation of understanding which features are peculiar of dimension two.

In this paper we will consider multi-phase covariant cloning transformations on "equatorial" states

$$
\left|\psi\left(\left\{\phi_{j}\right\}\right)\right\rangle=\frac{1}{\sqrt{d}}\left(|0\rangle+e^{i \phi_{1}}|1\rangle+e^{i \phi_{2}}|2\rangle+\ldots+e^{i \phi_{d-1}}|d-1\rangle\right),
$$

where the $\phi_{j}$ 's are independent phases in the interval $[0,2 \pi)$. An issue which recently has attracted interest in the literature is the possibility of achieving the cloning without the need of an ancilla - a so-called "economical" cloning 11. As we will see in the following, the multi-phase covariant cloning machines are indeed economical for $M=k d+N$ output copies, $k$ integer.

The paper is organized as follows. In Section III after introducing the notations and the basic definitions taken from Refs. [8, 10], we describe the general approach to covariant cloning maps of Ref. [12], and apply it to the case of $N \rightarrow M$ phase-covariant cloner. In Section [II] we give a brief formalization of the concept of "economical maps" by means of the Stinespring representation theorem for completely positive maps. In Section IV we explicitly find the optimal $1 \rightarrow M$ cloner for both single-qudit fidelity and global fidelities. In Section $\nabla$ we generalize all previous results to the case $N \rightarrow M$. Section $\nabla I$ concludes the paper with a comparison of fidelities in the various cases.

\section{PHASE-COVARIANT CLONING}

We want to derive the optimal $N \rightarrow M$ cloning transformations $\mathcal{C}$ that are covariant under the group of rotations of all the $d-1$ independent phases $\left\{\phi_{j}\right\}, \phi_{j} \in[0,2 \pi)$,

$$
U\left(\left\{\phi_{j}\right\}\right)=|0\rangle\left\langle 0\left|+\sum_{j=1}^{d-1}\right| j\right\rangle\langle j| e^{i \phi_{j}},
$$

\footnotetext{
*Electronic address: buscemi@fisicavolta.unipv.it

$\dagger$ Electronic address: dariano@unipv.it

‡Electronic address: chiara@unipv.it

$\S$ URL: http://www.qubit.it/
} 
where $\{|0\rangle,|1\rangle,|2\rangle \ldots|d-1\rangle\}$ represents a basis for the $d$-dimensional Hilbert space $\mathcal{H}$ of the system of a single copy. We will restrict the study of such maps to the set of the $N$-fold tensor product of generalized equatorial pure states

$$
U\left(\left\{\phi_{j}\right\}\right)\left|\psi_{0}\right\rangle \doteq\left|\psi\left(\left\{\phi_{j}\right\}\right)\right\rangle
$$

with $\left|\psi\left(\left\{\phi_{j}\right\}\right)\right\rangle$ given in Eq. (1). Here $\left|\psi_{0}\right\rangle$ is the equatorial superposition

$$
\left|\psi_{0}\right\rangle=d^{-1 / 2} \sum_{i}|i\rangle
$$

The choice $\phi_{0}=0$ is not restrictive, since an overall phase is negligible.

As argued in Ref. [8], we consider cloning maps for which the $N$-copy input state and the $M$-copy output state are both supported on the symmetric subspaces $\mathcal{H}_{+}^{\otimes N}$ and $\mathcal{H}_{+}^{\otimes M}$, respectively. We choose orthonormal basis in the symmetric subspace of the form

$$
\left|\left\{n_{i}\right\}\right\rangle \doteq\left|n_{0}, n_{1}, n_{2}, \ldots n_{d-1}\right\rangle=\frac{1}{\sqrt{N !}} \sum_{\{\pi\}} P_{\pi}^{(N)}|\underbrace{00 \ldots 0}_{n_{0}} \underbrace{11 \ldots 1}_{n_{1}} \cdots \underbrace{d-1 \ldots d-1}_{n_{d-1}}\rangle,
$$

where $P_{\pi}^{(N)}$ denotes the permutation operator of $N$ qubits, $n_{0}$ is the number of qudits in state $|0\rangle$, $n_{1}$ in state $|1\rangle$, and so on, with the constraint $\sum_{i=0}^{d-1} n_{i}=N$ for the input state, and, analogously, for the output state. In the whole paper we will consistently use letters $n$ 's for input and $m$ 's for output. The covariance condition for the cloning transformation $\mathcal{C}$ under the group of multi-phases rotations reads

$$
\mathcal{C}\left(U\left(\left\{\phi_{j}\right\}\right)^{\otimes N} \rho^{\otimes N} U^{\dagger}\left(\left\{\phi_{j}\right\}\right)^{\otimes N}\right)=U\left(\left\{\phi_{j}\right\}\right)^{\otimes M} \mathcal{C}\left(\rho^{\otimes N}\right) U^{\dagger}\left(\left\{\phi_{j}\right\}\right)^{\otimes M} .
$$

As proved in Ref. 12] the covariance condition can be conveniently studied in terms of the positive operator on $\mathcal{H}_{+}^{\otimes M} \otimes \mathcal{H}_{+}^{\otimes N}$

$$
R \doteq(\mathcal{C} \otimes \mathcal{I})(|\mathbb{1}\rangle\rangle\langle\langle\mathbb{1}|)
$$

where $\mathcal{I}$ is the identity map and $|\mathbb{1}\rangle\rangle$ is the non normalized maximally entangled vector in $\mathcal{H}_{+}^{\otimes N} \otimes \mathcal{H}_{+}^{\otimes N}$

$$
|\mathbb{1}\rangle\rangle=\sum_{\left\{n_{i}\right\}}\left|\left\{n_{i}\right\}\right\rangle \otimes\left|\left\{n_{i}\right\}\right\rangle
$$

The correspondence $\mathcal{C} \leftrightarrow R$ between completely positive maps and positive operators is one-to-one, and can be inverted as follows

$$
\mathcal{C}(O)=\operatorname{Tr}_{\mathcal{H}_{+}^{\otimes N}}\left[\left(\mathbb{1}_{\mathcal{H}_{+}^{\otimes M}} \otimes O^{T}\right) R\right]
$$

where $O^{T}$ denotes the transposition of the operator $O$ with respect to the orthonormal basis in Eq. (8). Notice that for the state $\left|\psi_{0}\right\rangle$ of Eq. (4) one has $\left(\left|\psi_{0}\right\rangle\left\langle\left.\psi_{0}\right|^{\otimes N}\right)^{T}=\left|\psi_{0}\right\rangle\left\langle\left.\psi_{0}\right|^{\otimes N}\right.\right.$ since $\left.\mid \psi_{0}\right\rangle$, by construction, has all real coefficients with respect to the basis in Eq. (8). The trace-preservation condition for $\mathcal{C}$ reads

$$
\operatorname{Tr}_{\mathcal{H}_{+}^{\otimes M}}[R]=\mathbb{1}_{\mathcal{H}_{+}^{\otimes N}}
$$

Following Ref. [12], the covariance property (6) rewrites as a commutation relation

$$
\left[R, U\left(\left\{\phi_{j}\right\}\right)^{\otimes M} \otimes U^{*}\left(\left\{\phi_{j}\right\}\right)^{\otimes N}\right]=0
$$

where the complex conjugated $U^{*}$ of $U$ is defined as the operator having as matrix elements the complex-conjugated matrix elements of $U$ with respect to the same orthonormal basis in Eq. (8). Eq. (11) in turn implies by Schur Lemma a block-form for $R$

$$
R=\bigoplus_{\left\{m_{j}\right\}} R_{\left\{m_{j}\right\}}
$$

where each set of values $\left\{m_{j}\right\}$ identifies a unique class of equivalent irreducible representations of $U\left(\left\{\phi_{j}\right\}\right)^{\otimes M} \otimes$ $U^{*}\left(\left\{\phi_{j}\right\}\right)^{\otimes N}$. The equivalent representations within each class can be conveniently written as

$$
\left\{\left|m_{0}+n_{0}, m_{1}+n_{1}, m_{2}+n_{2}, \ldots m_{d-1}+n_{d-1}\right\rangle\left|n_{0}, n_{1}, n_{2}, \ldots n_{d-1}\right\rangle\right\}_{\left\{n_{i}\right\}},
$$


with $\sum_{i=0}^{d-1} n_{i}=N$ and $\sum_{j=0}^{d-1} m_{j}=M-N$. The multi-index $\left\{n_{i}\right\}$ runs over all orthonormal vectors of the basis for $\mathcal{H}_{+}^{\otimes N}$ used in Eq. (8). With this notation, Eq. (12) becomes

$$
R=\sum_{\left\{m_{j}\right\}} \sum_{\left\{n_{i}^{\prime}\right\},\left\{n_{i}^{\prime \prime}\right\}} r_{\left\{n_{i}^{\prime}\right\},\left\{n_{i}^{\prime \prime}\right\}}^{\left\{m_{j}\right\}}\left|\left\{m_{j}\right\}+\left\{n_{i}^{\prime}\right\}\right\rangle\left\langle\left\{m_{j}\right\}+\left\{n_{i}^{\prime \prime}\right\}|\otimes|\left\{n_{i}^{\prime}\right\}\right\rangle\left\langle\left\{n_{i}^{\prime \prime}\right\}\right| .
$$

In the following, in order to evaluate the optimality of the map, we will use as figures of merit the single-qudit fidelity

$$
\operatorname{Tr}\left[\left(\left|\psi_{0}\right\rangle\left\langle\psi_{0}\left|\otimes \mathbb{1}^{M-1} \otimes\right| \psi_{0}\right\rangle\left\langle\left.\psi_{0}\right|^{\otimes N}\right) R\right]\right.
$$

and the global fidelity

$$
\operatorname{Tr}\left[\left(\left|\psi_{0}\right\rangle\left\langle\left.\psi_{0}\right|^{\otimes M+N}\right) R\right]\right.
$$

Notice that in deriving the last two equations we used the covariance property (3) of the input states, the reconstruction formula (9), the commutation property (11), and the cyclic invariance of the trace. Since each single contribution to the single-qudit fidelity (15) and to the global fidelity (16) is positive versus the indeces $\left\{n_{i}^{\prime}\right\}$ and $\left\{n_{i}^{\prime \prime}\right\}$, as we will show in the following (see Eqs. (36), (37), and (46), 13]), the block $R_{\left\{m_{j}\right\}}$ must have positive elements $r_{\left\{n_{i}^{\prime}\right\},\left\{n_{i}^{\prime \prime}\right\}} \geq 0$, with the off-diagonal ones as large as possible, i.e. $r_{\left\{n_{i}^{\prime}\right\},\left\{n_{i}^{\prime \prime}\right\}}^{\left\{m_{j}\right\}}=\sqrt{r_{\left\{n_{i}^{\prime}\right\},\left\{n_{i}^{\prime}\right\}}^{\left\{m_{j}\right\}}} \sqrt{r_{\left\{n_{i}^{\prime \prime}\right\},\left\{n_{i}^{\prime \prime}\right\}}^{\left\{m_{j}\right.}}$, [14]. This is equivalent to say that the blocks constituting the operator $R$ are actually rank-one blocks, namely

$$
R_{\left\{m_{j}\right\}} \propto\left|r_{\left\{m_{j}\right\}}\right\rangle\left\langle r_{\left\{m_{j}\right\}}\right|
$$

with

$$
\left|r_{\left\{m_{j}\right\}}\right\rangle=\sum_{\left\{n_{i}\right\}} r_{\left\{n_{i}\right\}}^{\left\{m_{j}\right\}}\left|\left\{m_{j}\right\}+\left\{n_{i}\right\}\right\rangle \otimes\left|\left\{n_{i}\right\}\right\rangle
$$

and, separately imposing condition (10) over every block, 15], $\operatorname{Tr}_{\mathcal{H}_{+}^{\otimes M}}\left[\left|r_{\left\{m_{j}\right\}}\right\rangle\left\langle r_{\left\{m_{j}\right\}}\right|\right]=\mathbb{1}_{\mathcal{H}_{+}^{\otimes N}}$, we get the final form for $R$

$$
R=\bigoplus_{\left\{m_{j}\right\}} p_{\left\{m_{j}\right\}}\left|r_{\left\{m_{j}\right\}}\right\rangle\left\langle r_{\left\{m_{j}\right\}}|, \quad| r_{\left\{m_{j}\right\}}\right\rangle=\sum_{\left\{n_{i}\right\}}\left|\left\{m_{j}\right\}+\left\{n_{i}\right\}\right\rangle \otimes\left|\left\{n_{i}\right\}\right\rangle,
$$

where $p_{\left\{m_{j}\right\}}$ are free parameters satisfying $p_{\left\{m_{j}\right\}} \geq 0$ and $\sum p_{\left\{m_{j}\right\}}=1$ in order to preserve normalization and positivity of $R$. This means that $R$ is a convex combination of orthogonal rank-one blocks.

In Sections [V] and $\nabla$ we will explicitly optimize the map starting from the $R$ operator in Eq. (19).

\section{ECONOMICAL MAPS}

Let $\mathcal{M}$ be a completely positive, trace-preserving map from states on $\mathcal{H}$ to states on $\mathcal{K}$. The Stinespring representation Theorem [16] says that for every completely positive trace-preserving map it is possible to find an auxiliary quantum system with Hilbert space $\mathcal{L}$ and an isometry $V$ from $\mathcal{H}$ to $\mathcal{K} \otimes \mathcal{L}, V^{\dagger} V=\mathbb{1}_{\mathcal{H}}$, such that

$$
\mathcal{M}(\rho)=\operatorname{Tr}_{\mathcal{L}}\left[V \rho V^{\dagger}\right]
$$

Starting from Eq. (20), it is always possible to construct a unitary interaction $U$ realizing $\mathcal{M}$ [17, 18]:

$$
\mathcal{M}(\rho)=\operatorname{Tr}_{\mathcal{L}}\left[U(\rho \otimes|a\rangle\langle a|) U^{\dagger}\right]
$$

where $|a\rangle$ is a fixed pure state of a second auxiliary quantum system, say $\mathcal{L}^{\prime}$, such that $\mathcal{H} \otimes \mathcal{L}^{\prime}=\mathcal{K} \otimes \mathcal{L}$. The Hilbert spaces $\mathcal{L}$ and $\mathcal{L}^{\prime}$ are generally different, and actually play different physical roles.

We define a trace-preserving completely positive map $\mathcal{M}$ to be economical if and only if it admits a unitary form $U$ as

$$
\mathcal{M}(\rho)=U(\rho \otimes|a\rangle\langle a|) U^{\dagger}
$$


namely, if and only if the map can be physically realized without discarding resources. We can simply prove that the only maps admitting an economical unitary implementation $U$ as in Eq. 22) are precisely those for which

$$
\mathcal{M}(\rho)=V \rho V^{\dagger}
$$

for an isometry $V, V^{\dagger} V=\mathbb{1}$. In fact, $U\left(\mathbb{1}_{\mathcal{H}} \otimes|a\rangle\right)$ is an isometry from $\mathcal{H}$ to $\mathcal{K} \otimes \mathcal{L}$, since $\left(\mathbb{1}_{\mathcal{H}} \otimes\langle a|\right) U^{\dagger} U\left(\mathbb{1}_{\mathcal{H}} \otimes|a\rangle\right)=\mathbb{1}_{\mathcal{H}}$. On the other hand, from Eq. 23] via Gram-Schmidt one can extend any isometry $V$ from $\mathcal{H}$ to $\mathcal{K} \otimes \mathcal{L}$ to a unitary $U$ on the same output space, and write it in the form $V=U\left(\mathbb{1}_{\mathcal{H}} \otimes|a\rangle\right)$ for unit vector $|a\rangle \in \mathcal{L}^{\prime}$, with $\mathcal{H} \otimes \mathcal{L}^{\prime}=\mathcal{K} \otimes \mathcal{L}$. For a detailed discussion about the explicit construction procedures, see Ref. [18].

Allowing classical resources "for free", a map should be defined as economical also in the case in which it admits a random-economical realization as

$$
\mathcal{M}(\rho)=\sum_{i} p_{i} U_{i}(\rho \otimes|a\rangle\langle a|) U_{i}^{\dagger}
$$

where $p_{i} \geq 0, \sum_{i} p_{i}=1$. Using the same fixed ancilla state $|a\rangle$ for all indeces $i$ is not a loss of generality, since in constructing the operators $U_{i}$ 's there is always freedom in the choice of the vector $|a\rangle$. According to this more general definition, all economical maps can always be written as a randomization of Eq. (23):

$$
\mathcal{M}(\rho)=\sum_{i} p_{i} V_{i} \rho V_{i}^{\dagger}
$$

\section{OPTIMAL $1 \rightarrow M$ CLONING}

The fidelity of the reduced output state $\operatorname{Tr}_{M-1}\left[\mathcal{C}\left(\left|\psi\left(\left\{\phi_{j}\right\}\right)\right\rangle\left\langle\psi\left(\left\{\phi_{j}\right\}\right)\right|\right)\right]$ with respect to the input state $\left|\psi\left(\left\{\phi_{j}\right\}\right)\right\rangle\left\langle\psi\left(\left\{\phi_{j}\right\}\right)\right|$ is given by

$$
\operatorname{Tr}\left[\left(\left|\psi_{0}\right\rangle\left\langle\psi_{0}\left|\otimes \mathbb{1}^{M-1} \otimes\right| \psi_{0}\right\rangle\left\langle\psi_{0}\right|\right) R\right]
$$

In the case of $1 \rightarrow M$ cloning, the $R$ operator in Eq. (19) has the following structure:

$$
R=\bigoplus_{\left\{m_{j}\right\}} p_{\left\{m_{j}\right\}}\left|r_{\left\{m_{j}\right\}}\right\rangle\left\langle r_{\left\{m_{j}\right\}}|, \quad| r_{\left\{m_{j}\right\}}\right\rangle=\sum_{i}\left|m_{0}, m_{1}, \ldots, m_{i}+1, \ldots\right\rangle \otimes|i\rangle,
$$

with $\sum_{j} m_{j}=M-1$, whence the form of the summands:

$$
\begin{aligned}
\operatorname{Tr} & {\left[\left(\left|\psi_{0}\right\rangle\left\langle\psi_{0}\left|\otimes \mathbb{1}^{M-1} \otimes\right| \psi_{0}\right\rangle\left\langle\psi_{0}\right|\right)\left|m_{0}+1, m_{1}, m_{2}, \ldots m_{d-1}\right\rangle\left\langle m_{0}, m_{1}+1, m_{2}, \ldots m_{d-1}|\otimes| 0\right\rangle\langle 1|\right] } \\
& =\frac{1}{d^{2}} \frac{(M-1) !}{m_{0} ! m_{1} ! \ldots m_{d-1} !} \sqrt{\frac{\left(m_{0}+1\right) ! m_{1} ! \ldots m_{d-1} !}{M !} \frac{m_{0} !\left(m_{1}+1\right) ! \ldots m_{d-1} !}{M !}}=\frac{1}{M d^{2}} \sqrt{\left(m_{0}+1\right)\left(m_{1}+1\right)}
\end{aligned}
$$

The final contribution to the partial fidelity due to the set of equivalent representations labeled by $\left\{m_{j}\right\}$ is

$$
F_{\left\{m_{j}\right\}}=\operatorname{Tr}\left[\left(\left|\psi_{0}\right\rangle\left\langle\psi_{0}\left|\otimes \mathbb{1}^{M-1} \otimes\right| \psi_{0}\right\rangle\left\langle\psi_{0}\right|\right) R_{\left\{m_{j}\right\}}\right]=\frac{p_{\left\{m_{j}\right\}}}{d^{2}}\left[d+\frac{1}{M} \sum_{i \neq k} \sqrt{\left(m_{i}+1\right)\left(m_{k}+1\right)}\right] .
$$

The projector $\left|r_{\left\{m_{j}\right\}}\right\rangle\left\langle r_{\left\{m_{j}\right\}}\right|$ that contributes most to the fidelity is the one that maximizes the quantity $\sum_{i \neq k} \sqrt{\left(m_{i}+1\right)\left(m_{k}+1\right)}$, with the constraint $\sum_{i=0}^{d-1} m_{i}=M-1$. In the case

$$
M=d k+1, \quad k \in \mathbb{N}
$$

the optimization gives the simple result $m_{i}=k$ for all $i$. The $1 \rightarrow(k d+1)$ optimal phase-covariant cloning machine is then completely described by the rank-one positive operator $R=\left|r_{\{k\}}\right\rangle\left\langle r_{\{k\}}\right|$. The Kraus form of the optimal map is then reconstructed as

$$
\mathcal{C}(\rho)=\operatorname{Tr}_{\mathcal{H}}\left[\left(\mathbb{1}_{\mathcal{H}_{+}^{\otimes M}} \otimes \rho^{T}\right) R\right]=V \rho V^{\dagger}
$$


where $V: \mathcal{H} \rightarrow \mathcal{H}_{+}^{\otimes M}$ is the isometry, i.e. $V^{\dagger} V=\mathbb{1}$, defined as follows:

$$
V|i\rangle=\left|m_{0}=k, m_{1}=k, \ldots, m_{i}=k+1, \ldots\right\rangle .
$$

The fact that the Kraus operator describing the map is isometrical - a consequence of $R$ being rank-oneautomatically guarantees that no additional ancillae (other than the $M-1$ blank states) are needed in order to unitarily realize the cloning transformation $\mathcal{C}$.

From Eq. (29) one obtains the single-qudit fidelity of our multi-phase-covariant economical cloning machine from one to $M=k d+1$ copies

$$
F_{\mathcal{C}}(1, M=k d+1)=\frac{1}{d}+\frac{(d-1)(M+d-1)}{M d^{2}} .
$$

Notice that the above result, in the limit $M \rightarrow \infty$, is consistent with the fidelity of optimal phase estimation on a single qudit as worked out in Ref. [10].

An important remark that remains to be stressed is that the value of $\left\{m_{j}\right\}$ maximizing the single-qudit fidelity (29) maximizes the total fidelity as well. In fact, the total fidelity of the $\left\{m_{j}\right\}$-th block is given by

$$
\begin{gathered}
\left.p_{\left\{m_{j}\right\}} \sum_{i j} \operatorname{Tr}\left[\left|\psi_{0}\right\rangle\left\langle\left.\psi_{0}\right|^{\otimes(M+1)} \mid \ldots, m_{i}+1, \ldots\right\rangle\left\langle\ldots, m_{j}+1, \ldots|\otimes| i\right\rangle\langle j|\right]=p_{\left\{m_{j}\right\}} \sum_{i}\left|\left\langle\psi_{0}^{\otimes(M+1)} \mid \ldots, m_{i}+1, \ldots\right\rangle\right| i\right\rangle\left.\right|^{2} \\
=\frac{p_{\left\{m_{j}\right\}}}{d^{M+1}} \sum_{i} \frac{M !}{m_{0} ! \ldots\left(m_{i}+1\right) ! \ldots}=\frac{p_{\left\{m_{j}\right\}}}{d^{M+1}} \sum_{i}\left(\begin{array}{c}
M \\
m_{0} ; \cdots ; m_{i}+1 ; \ldots
\end{array}\right)
\end{gathered}
$$

where, in the last line, we used the standard notation for multinomial coefficients with the implicit constraints $\sum_{j} m_{j}=M-1$. In order to maximize the value of the multinomial coefficient, the vector $\left\{m_{j}\right\}$ has to be as "flat" as possible, namely, with all entries as close as possible to each other. The situation in which the solution is unique and given by $m_{i}=k$ for all $i$ is the same as in Eq. (30). This means that the single-qudit fidelity optimization procedure provides the same result as the total fidelity optimization, and the map written in Eqs. (31) and (32) is optimal in both approaches.

\section{OPTIMAL $N \rightarrow M$ CLONING}

In the general case of arbitrary values for $N$ and $M$, the single-qudit fidelity is obtained by summing up contributions of the form

$$
\operatorname{Tr}\left[\left|\psi_{0}\right\rangle\left\langle\psi_{0}\left|\otimes \mathbb{1}^{\otimes(M-1)} \otimes\right| \psi_{0}\right\rangle\left\langle\left.\psi_{0}\right|^{\otimes N} \mid\left\{m_{j}\right\}+\left\{n_{j}^{\prime}\right\}\right\rangle\left\langle\left\{m_{j}\right\}+\left\{n_{j}^{\prime \prime}\right\}|\otimes|\left\{n_{j}^{\prime}\right\}\right\rangle\left\langle\left\{n_{j}^{\prime \prime}\right\}\right|\right]
$$

because of the block-form (19) of the $R$ operator. Before getting into the explicit calculation for the partial fidelity, it is possible to somehow simplify the problem by noticing that the presence of $\left|\psi_{0}\right\rangle\left\langle\psi_{0}\right| \otimes \mathbb{1}^{\otimes(M-1)}$ in Eq. (35) restricts the evaluation of the fidelity to those blocks for which the $M$-particles states differ at most for a single-particle state.

The diagonal contributions to $F_{\left\{m_{j}\right\}}$ (as before, the single-qudit fidelity calculated only for the $\left\{m_{j}\right\}$-th block) are then proportional to (apart from the probability $p_{\left\{m_{j}\right\}}$ )

$$
\begin{aligned}
\operatorname{Tr} & {\left[\left|\psi_{0}\right\rangle\left\langle\psi_{0}\left|\otimes \mathbb{1}^{\otimes(M-1)} \otimes\right| \psi_{0}\right\rangle\left\langle\left.\psi_{0}\right|^{\otimes N} \mid \ldots, m_{i}+n_{i}+1, \ldots\right\rangle\left\langle\ldots, m_{i}+n_{i}+1, \ldots|\otimes| \ldots, n_{i}+1, \ldots\right\rangle\left\langle\ldots, n_{i}+1, \ldots\right|\right] } \\
& =\frac{1}{d^{N}} \frac{N !}{n_{0} ! \ldots\left(n_{i}+1\right) ! \ldots} \frac{1}{d} \frac{\left(m_{0}+n_{0}\right) ! \ldots\left(m_{i}+n_{i}+1\right) ! \ldots}{M !} \frac{M !}{\left(m_{0}+n_{0}\right) ! \ldots\left(m_{i}+n_{i}+1\right) ! \ldots} \\
& =\frac{1}{d^{N+1}} \frac{N !}{n_{0} ! \ldots\left(n_{i}+1\right) ! \ldots}
\end{aligned}
$$

where for sake of simplicity in the last equation the notation was slightly modified with $\sum_{i} n_{i}=N-1$ while, again, 
$\sum_{j} m_{j}=M-N$. The off-diagonal terms are

$$
\begin{aligned}
\operatorname{Tr} & {\left[\left|\psi_{0}\right\rangle\left\langle\psi_{0}\left|\otimes \mathbb{1}^{\otimes(M-1)} \otimes\right| \psi_{0}\right\rangle\left\langle\left.\psi_{0}\right|^{\otimes N} \mid \ldots, m_{i}+n_{i}+1, \ldots\right\rangle\left\langle\ldots, m_{j}+n_{j}+1, \ldots|\otimes| \ldots, n_{i}+1, \ldots\right\rangle\left\langle\ldots, n_{j}+1, \ldots\right|\right] } \\
= & \operatorname{Tr}\left[\left|\psi_{0}\right\rangle\left\langle\psi_{0}\left|\otimes \mathbb{1}^{\otimes(M-1)}\right| \ldots, m_{i}+n_{i}+1, \ldots\right\rangle\left\langle\ldots, m_{j}+n_{j}+1, \ldots\right|\right] \\
& \times \frac{1}{d^{N}} \sqrt{\frac{n_{0} ! \ldots\left(n_{i}+1\right) ! \ldots}{N !}} \sqrt{\frac{n_{0} ! \ldots\left(n_{j}+1\right) ! \ldots}{N !}} \frac{N !}{n_{0} ! \ldots\left(n_{i}+1\right) ! \ldots} \frac{N !}{n_{0} ! \ldots\left(n_{j}+1\right) ! \ldots} \\
= & \frac{1}{d^{N+1}} \frac{N !}{n_{0} ! \ldots n_{i} ! \ldots n_{j} ! \ldots} \sqrt{\frac{1}{\left(n_{i}+1\right)\left(n_{j}+1\right)}} \sqrt{\frac{\left(m_{0}+n_{0}\right) ! \ldots\left(m_{i}+n_{i}+1\right) ! \ldots}{M !}} \\
& \times \sqrt{\frac{\left(m_{0}+n_{0}\right) ! \ldots\left(m_{j}+n_{j}+1\right) ! \ldots}{M !}} \frac{(M-1) !}{\left(m_{0}+n_{0}\right) ! \ldots\left(m_{i}+n_{i}\right) ! \ldots\left(m_{j}+n_{j}\right) ! \ldots} \\
= & \frac{1}{M d^{N+1}} \frac{N !}{n_{0} ! \ldots n_{i} ! \ldots n_{j} ! \ldots} \sqrt{\frac{\left(m_{i}+n_{i}+1\right)\left(m_{j}+n_{j}+1\right)}{\left(n_{i}+1\right)\left(n_{j}+1\right)}} .
\end{aligned}
$$

At the end, the single-qudit fidelity is the sum of contributions of the form

$$
F_{\left\{m_{j}\right\}}=\frac{p_{\left\{m_{j}\right\}}}{d^{N+1}} \sum_{\left\{n_{j}\right\}}\left[\sum_{i} \frac{N !}{n_{0} ! \ldots\left(n_{i}+1\right) ! \ldots}+\frac{1}{M} \sum_{i \neq j} \frac{N !}{n_{0} ! \ldots n_{i} ! \ldots n_{j} ! \ldots} \sqrt{\frac{\left(m_{i}+n_{i}+1\right)\left(m_{j}+n_{j}+1\right)}{\left(n_{i}+1\right)\left(n_{j}+1\right)}}\right] .
$$

As done before for the $1 \rightarrow M$ cloning, in order to find the block of $R$ realizing the optimal map, we have to maximize the off-diagonal quantity

$$
\sum_{i \neq j} \sqrt{\frac{\left(m_{i}+n_{i}+1\right)\left(m_{j}+n_{j}+1\right)}{\left(n_{i}+1\right)\left(n_{j}+1\right)}}
$$

with the constraints $\sum_{i} n_{i}=N-1$ and $\sum_{j} m_{j}=M-N$. The maximization of fidelity corresponds to maximize the quantity in Eq. (39) versus the variables $m_{i}$ 's. Since the variables $n_{i}$ 's are summed up in Eq. (38), then the fidelity is invariant under their permutation. Therefore the evaluation of the maximum of the quantity (39) resorts to maximize it for equal $n_{i}$ 's, whence also all $m_{i}$ 's will be equal, $m_{i}=m_{*} \doteq(M-N) / d, \forall i$. Generally, in this way one obtains a non integer value of $m_{*}$, while the maximum for integer $m_{i}$ 's is very degenerate, since the maximum will be obtained for unequal $m_{i}$ 's in place of a common fractional value. This leads to many blocks for $R$ contributing in the same way to the optimal map, which makes the evaluation very complicate. On the other hand, the evaluation simplifies greatly when the maximum is achieved for integer $m_{*}=k$, and this corresponds to the following relation between $N$ and $M$

$$
M=k d+N
$$

Hence, the optimal phase-covariant $N \rightarrow(N+k d)$ cloning map is described by the rank-one operator

$$
R=\left|r_{\{k\}}\right\rangle\left\langle r_{\{k\}}\right|,
$$

where

$$
\left|r_{\{k\}}\right\rangle=\sum_{\left\{n_{j}\right\}}\left|k+n_{0}, \ldots, k+n_{i}, \ldots\right\rangle_{M}\left|n_{0}, \ldots, n_{i} \ldots\right\rangle_{N}, \quad \sum_{j} n_{j}=N,
$$

and its single-qudit fidelity is given by

$$
F_{\mathcal{C}}(N, M=k d+N)=\frac{1}{d}+\frac{1}{M d^{N+1}} \sum_{\left\{n_{j}\right\}} \sum_{i \neq j} \frac{N !}{n_{0} ! \ldots n_{i} ! \ldots n_{j} ! \ldots} \sqrt{\frac{\left(n_{i}+k+1\right)\left(n_{j}+k+1\right)}{\left(n_{i}+1\right)\left(n_{j}+1\right)}}, \quad \sum_{j} n_{j}=N-1 .
$$

Notice that being $R$ rank-one, the optimal map derived here is again described by only one isometric Kraus operator $V: \mathcal{H}_{+}^{\otimes N} \rightarrow \mathcal{H}_{+}^{\otimes M}$

$$
\mathcal{C}\left(\rho^{\otimes N}\right)=\operatorname{Tr}_{\mathcal{H}_{+}^{\otimes N}}\left[\left(\mathbb{1}_{\mathcal{H}_{+}^{\otimes M}} \otimes\left(\rho^{\otimes N}\right)^{T}\right)\left|r_{\{k\}}\right\rangle\left\langle r_{\{k\}}\right|\right]=V \rho^{\otimes N} V^{\dagger}, \quad V^{\dagger} V=\mathbb{1}^{\otimes N},
$$


where the isometry $V$ acts as follows:

$$
V\left|n_{0}, n_{1}, \ldots, n_{i}, \ldots\right\rangle_{N}=\left|n_{0}+k, n_{1}+k, \ldots, n_{i}+k, \ldots\right\rangle_{M}
$$

Similarly to the case $1 \rightarrow(k d+1)$, the fact that the optimal $N \rightarrow(N+k d)$ cloning map is isometrical implies that no additional ancilla is needed to unitarily realize the map other than the $M-N$ blank copies, and (45) is again an economical cloning machine.

As in Section IV it is possible to prove that the value of $\left\{m_{j}\right\}$ maximizing the single-qudit fidelity maximizes the total fidelity as well

$$
\begin{aligned}
& \sum_{\left\{m_{j}\right\}} p_{\left\{m_{j}\right\}} \sum_{\left\{n_{j}^{\prime}\right\},\left\{n_{j}^{\prime \prime}\right\}} \operatorname{Tr}\left[\left|\psi_{0}\right\rangle\left\langle\left.\psi_{0}\right|^{\otimes(M+N)} \mid\left\{m_{j}\right\}+\left\{n_{j}^{\prime}\right\}\right\rangle\left\langle\left\{m_{j}\right\}+\left\{n_{j}^{\prime \prime}\right\}|\otimes|\left\{n_{j}^{\prime}\right\}\right\rangle\left\langle\left\{n_{j}^{\prime \prime}\right\}\right|\right] \\
= & \left.\sum_{\left\{m_{j}\right\}} p_{\left\{m_{j}\right\}} \sum_{\left\{n_{j}\right\}}\left|\left\langle\psi_{0}^{\otimes(M+N)} \mid m_{0}+n_{0}, m_{1}+n_{1}, \ldots\right\rangle\right| n_{0}, n_{1}, \ldots\right\rangle\left.\right|^{2} \\
= & \frac{1}{d^{M+N}} \sum_{\left\{m_{j}\right\}} p_{\left\{m_{j}\right\}} \sum_{\left\{n_{j}\right\}}\left(\begin{array}{c}
M \\
m_{0}+n_{0} ; m_{1}+n_{1} ; \cdots
\end{array}\right)\left(\begin{array}{c}
N \\
n_{0} ; n_{1} ; \cdots
\end{array}\right),
\end{aligned}
$$

with the usual constraints $\sum_{i} n_{i}=N$ and $\sum_{j} m_{j}=M-N$ implicit in the multinomial notation. Following the argument of the previous Section, it is clear that the map in Eqs. (44) and 45) maximizing the single-qudit fidelity (38), also maximizes the global fidelity (46).

As already noticed in the previous Section for $N=1$, the fidelity [43), in the limit of an infinite number of output copies, namely $k \rightarrow \infty$, takes the form (in the limit, $M \approx k d$ )

$$
F_{\mathrm{pe}}(N)=\frac{1}{d}+\frac{1}{d^{N+2}} \sum_{\left\{n_{i}\right\}} \sum_{i \neq j} \frac{N !}{n_{0} ! \ldots} \frac{1}{\sqrt{\left(n_{i}+1\right)\left(n_{j}+1\right)}} .
$$

The above expression coincides with the fidelity of optimal multi-phase estimation on equatorial qudits derived in Ref. [10].

\section{CONCLUSIONS}

We have addressed the problem of optimal phase-covariant cloning with multiple phases for qudits, with arbitrary number of input copies $N$ and output copies $M$. The optimization greatly simplifies for values of $M$ and $N$ related as $M=k d+N$, with $k$ integer. The cloning maps are optimal for both global and single-qudit fidelity. The map is achieved by an economical cloning machine, which works without ancilla. We have evaluated the asymptotic behaviour of the fidelity for large $M$, and recovered the fidelity of optimal multi-phase estimation [10]. In Figs. 1] 2] and [3] it is possible to compare the single-qudit fidelities of multi-phase covariant and universal covariant $1 \rightarrow M$ cloning machines. Increasing $M$ the fidelities tend to the limit of optimal phase estimation and state estimation fidelity, respectively. Increasing the dimension $d$ of the Hilbert space, the quality of the clones gets worse, as plotted in Fig. 4 Actually, for fixed $N$ and $M$, the single-qudit fidelity of the cloner goes to zero with $d^{-1}$, as turns out from Eq. (43). On the other hand, for fixed $M$, the fidelity saturates to one as $N$ gets close to $M$, in both multi-phase and universal covariant frameworks, as one can see in Figs. 5] 6] and 7] As general remark, notice that, increasing the dimension of the input system for fixed $N$ and $M$, the fidelities of multi-phase and universal cloners become closer to each other.

\section{ACKNOWLEDGMENTS}

This work has been jointly funded by the EC under the programs ATESIT (Contract No. IST-2000-29681), SECOQC (Contract No. IST-2003-506813) and INFM PRA-CLON.

[1] W. K. Wootters and W. H. Zurek, Nature (London) 299, 802 (1982).

[2] H. P. Yuen, Phys. Lett. A 113405 (1986).

[3] V. Bužek and M. Hillery, Phys. Rev. A 54, 1844 (1996). 


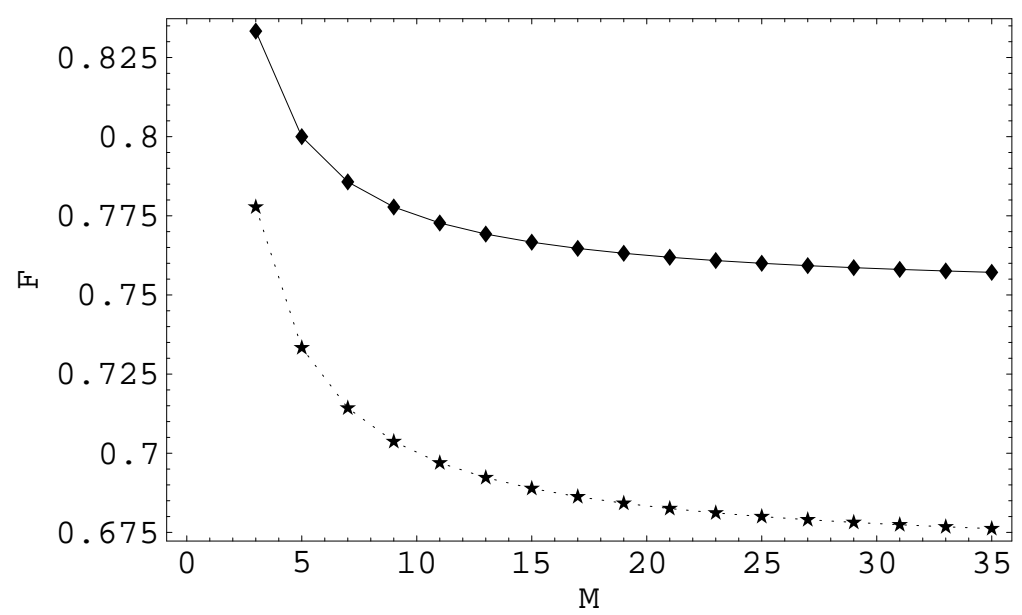

FIG. 1: Single-qubit fidelity for $1 \rightarrow M$ cloning: phase-covariant (continuous line); universal (dotted line). The numerical results are equal to those in Refs. [5] and [8].

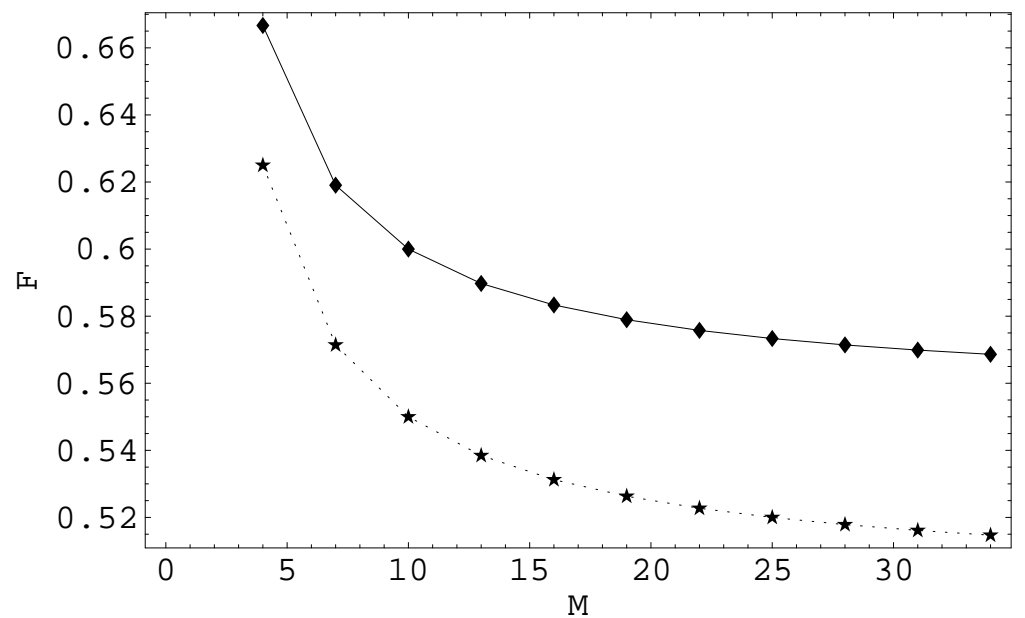

FIG. 2: Single-qutrit fidelity for $1 \rightarrow M$ cloning: multi-phase (continuous line); universal (dotted line).

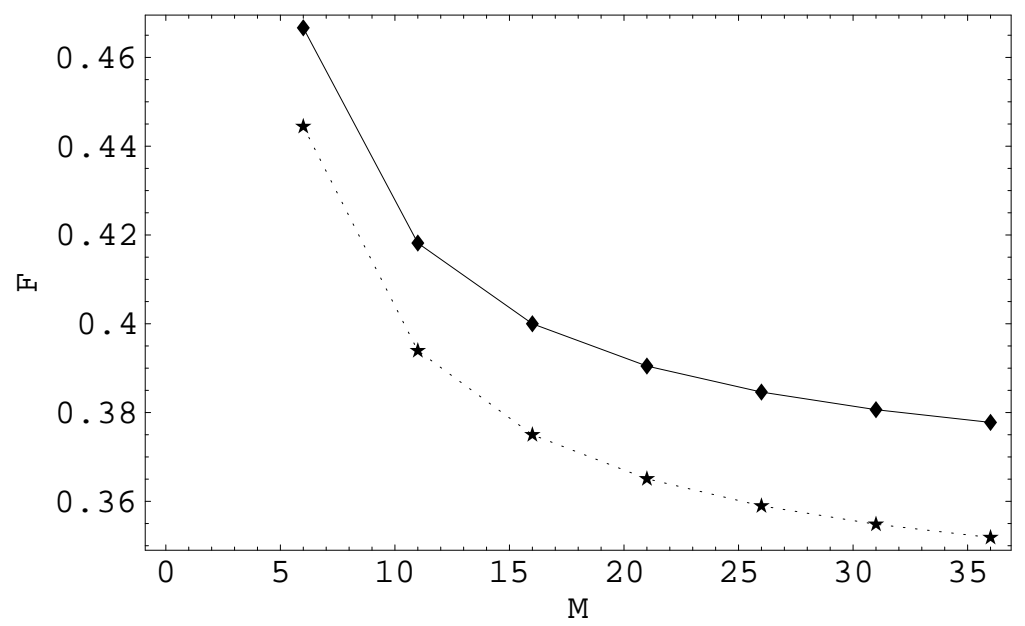

FIG. 3: Single-qudit, $d=5$, fidelity for $1 \rightarrow M$ cloning: multi-phase (continuous line); universal (dotted line). 


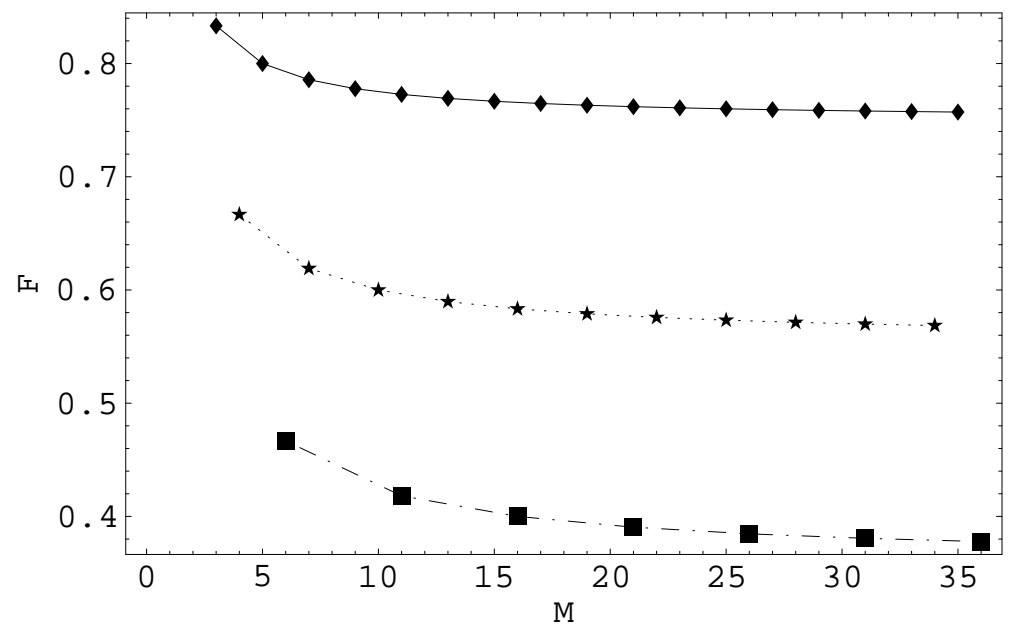

FIG. 4: Comparison between single-qudit fidelities for multi-phase covariant $1 \rightarrow M$ cloning: $d=2$ (continuous line); $d=3$ (dotted line); $d=5$ (dash dotted line). The three curves for large $M$ approach the fidelity of optimal phase estimation over one copy, namely $F=3 / 4$ for qubits, $F=5 / 9$ for qutrits, and $F=9 / 25$ for $d=5$.

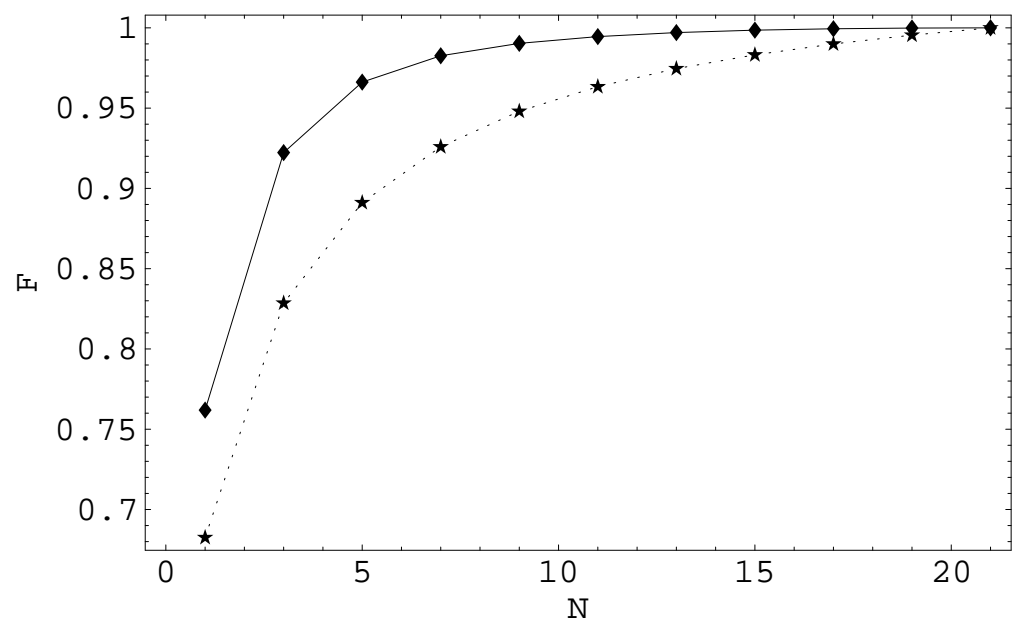

FIG. 5: Saturation of single-qubit fidelity for phase-covariant (continuous line) and universal (dotted line) $N \rightarrow M$ cloning machines versus $N$, for $M=21$.

[4] N. Gisin and S. Massar, Phys. Rev. Lett. 79, 2153 (1997).

[5] R. F. Werner, Phys. Rev. A 58, 1827 (1998).

[6] C. H. Bennet and G. Brassard in Proceedings of the IEEE International Conference of Computers, Systems and Signal Processing, (Bangalore, India 1984), pag. 175.

[7] D. Bruß, M. Cinchetti, G. M. D'Ariano, and C. Macchiavello, Phys. Rev. A 62012302 (2000).

[8] G. M. D'Ariano and C. Macchiavello, Phys. Rev. A 67, 042306 (2003).

[9] H. Fan, H. Imai, K. Matsumoto, and X.-B. Wang, Phys. Rev. A 67, 022317 (2003).

[10] C. Macchiavello, Phys. Rev. A 67, 062302 (2003).

[11] C.-S. Niu and R. B. Griffiths, Phys. Rev. A 60, 2764 (1999); T. Durt and J. Du, quant-ph/0309072

[12] G. M. D'Ariano and P. Lo Presti, Phys. Rev. A 64042308 (2001).

[13] More precisely, the single-qudit fidelity (15) and the global fidelity (16) are linear functionals in $r_{\left\{n_{i}^{\prime}\right\},\left\{n_{i}^{\prime \prime}\right\}}^{\{\text {of the form }}$ $\sum_{\left\{m_{j}\right\}} \sum_{\left\{n_{i}^{\prime}\right\},\left\{n_{i}^{\prime \prime}\right\}} a_{\left\{n_{i}^{\prime}\right\},\left\{n_{i}^{\prime \prime}\right\}}^{\left\{m_{j}\right\}} r_{\left\{n_{i}^{\prime}\right\},\left\{n_{i}^{\prime \prime}\right\}}^{\left\{m_{j}\right\}}$ with positive $a_{\left\{n_{i}^{\prime}\right\},\left\{n_{i}^{\prime \prime}\right\}}^{\left\{m_{j}\right\}}$.

[14] This bound comes from a Cauchy-Schwartz inequality $|(u, v)| \leq \sqrt{(u, u)} \sqrt{(v, v)}$ applied to the inner product induced by the positive matrix $R$, i. e. $(u, v) \doteq\langle u|R| v\rangle$.

[15] Actually one should impose condition (10) over all $R$. In the following, however, we will be able to single out only one optimal block of $R$. Thus, in this particular case, we are allowed to work with simpler coefficients without loss of generality. 


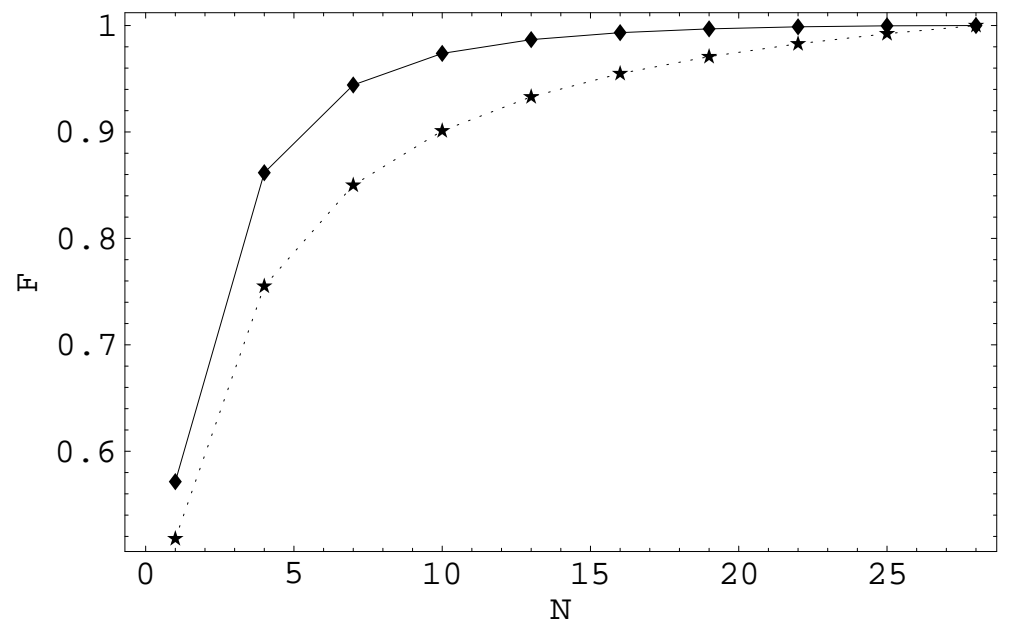

FIG. 6: Saturation of single-qutrit fidelity for multi-phase (continuous line) and universal (dotted line) $N \rightarrow M$ cloning machines versus $N$, for $M=28$.

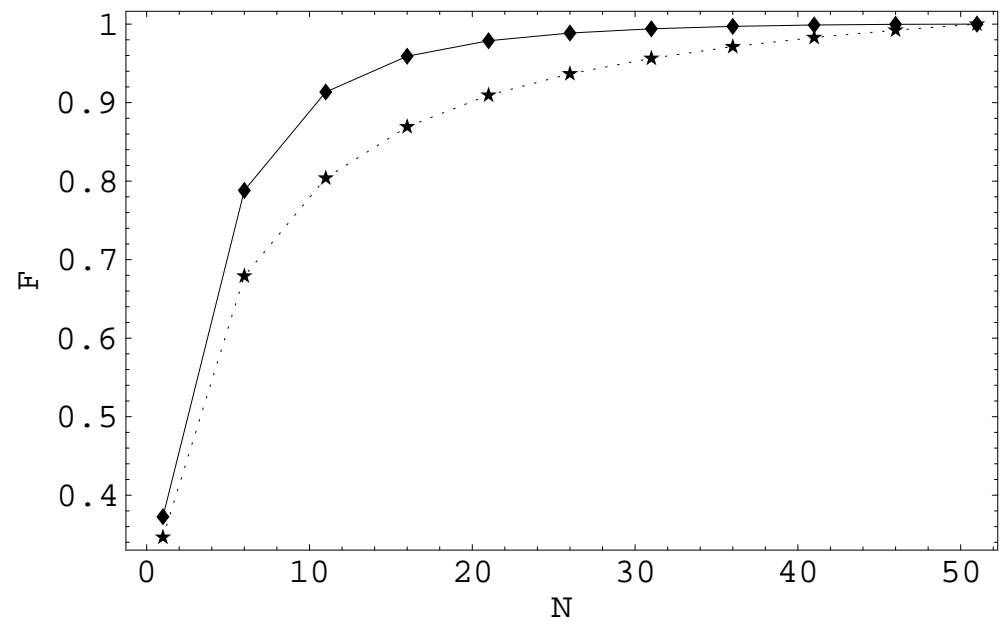

FIG. 7: Saturation of single-qudit, $d=5$, fidelity for multi-phase (continuous line) and universal (dotted line) $N \rightarrow M$ cloning machines versus $N$, for $M=51$.

[16] W. F. Stinespring, Proc. Am. Math. Soc. 6, 211 (1955).

[17] K. Kraus, in Foundations of Quantum Mechanics and Ordered Linear Spaces, Vol. 29 of Lecture Notes in Physics, 206 (Springer-Verlag, Berlin, Heidelberg, New York, 1973).

[18] F. Buscemi, G. M. D'Ariano, and M. F. Sacchi, Phys. Rev. A 68, 042113 (2003). 